AperTO - Archivio Istituzionale Open Access dell'Università di Torino

Prophylactic cranial irradiation in extensive disease small cell lung cancer: An endless debate

This is a pre print version of the following article:

Original Citation:

Availability:

This version is available http://hdl.handle.net/2318/1729967

since $2020-02-22 T 13: 34: 31 Z$

Published version:

DOI:10.1016/j.critrevonc.2019.08.010

Terms of use:

Open Access

Anyone can freely access the full text of works made available as "Open Access". Works made available under a Creative Commons license can be used according to the terms and conditions of said license. Use of all other works requires consent of the right holder (author or publisher) if not exempted from copyright protection by the applicable law. 
PROPHYLACTIC CRANIAL IRRADIATION IN EXTENSIVE STAGE SMALL CELL LUNG CANCER: WHAT IS THE CURRENT EVIDENCE AND WHAT REALLY MATTERS TO PATIENTS?

Authors and affiliations

Picardi $\mathrm{C}^{1}$, Banna $\mathrm{GL}^{2}$, Di Maio $\mathrm{M}^{3}$ Caparrotti $\mathrm{F}^{1}$, Addeo $\mathrm{A}^{4}$

1) Department of Radiation Oncology,

2) Department of Oncology, Cannizzaro Hospital, Catania

3) Department of Oncology, Ordine Mauriziano Hospital, University of Turin, Turin.

4) Department of Oncology, University Hospital of Geneva

Corresponding author:

Alfredo Addeo, Geneva University Hospital.

Rue Gabrielle-Perret-Gentil 4, 1205

Email alfredo.addeo@hcuge.ch

Tel +41784022664

Keywords: ED-SCLC, PCI, Brain metastases 


\section{OPINION STATEMENT:}

Extensive disease Small cell lung cancer (ED-SCLC) represents a very aggressive malignancy in which brain metastases are quite common. Clinical trials on prophylactic cranial irradiation (PCl) have showed a clear decrease in the risk of developing brain metastases but conflicting results regarding a possible survival advantage.

In particular the JCOG study didn't show any survival benefit in favour of $\mathrm{PCl}$ compared to brain MRI monitoring thus this has led to an important shifting in oncology practice. By review extensively literature on the role and importance of $\mathrm{PCl}$ in ED-SCLC it emerges clearly that PCI could still pllay a crucial role in the treatment of ED-SCLC. Given the conflicting results in term of OS, empowering the patient, by giving him-her the appropriate information is the way forward to offer a correct patient-related treatment.

\section{INTRODUCTION}

Small-cell lung cancer (SCLC) represents nearly $13 \%$ of all newly diagnosed lung cancers. Most patients present with extensive disease (ED) and, without treatment, median survival remains poor, ranging from 2 to 4 months [1]. At diagnosis, at least $18 \%$ of SCLC patients already present brain metastases (BM), the rate could increase up to $25 \%$ with more accurate brain imaging such as magnetic resonance imaging (MRI). Furthermore, the incidence of BM increases considerably during the course of the disease, reaching $80 \%$ in patients surviving 2 years after diagnosis [2]. The development of BM can be associated with severe neurological symptoms and significant impairment of health-related quality of life, with a median overall survival (OS) lower than 6 months. The blood-brain barrier is poorly penetrated by most of the anticancer drugs, making systemic treatment slightly ineffective to prevent such a 
complication. One landmark achievement to prevent the development of BM was the introduction of prophylactic cranial irradiation $(\mathrm{PCl})$ more than 40 years ago [3]. Since the publication of a meta-analysis in 1999 [4], $\mathrm{PCl}$ has been recommended as standard of care in SCLC patients responding to initial systemic treatment. This meta-analysis included 987 patients: and the use of $\mathrm{PCl}$ confirmed an absolute increase in the 3-year OS rate of $5.4 \%$, along with a significant decrease of BMs incidence (from $58.6 \%$ to $33.3 \%$ after 3 years). Even if patients with ED were only $15 \%$ of the whole population, the majority had limited disease (LD), the observed benefit was similar in the two subgroups.

In 2007, the European Organisation for Research and Treatment of Cancer (EORTC) published the results of a study about the role of PCI in ED-SCLC patients who had responded to chemotherapy [5]. $\mathrm{PCl}$ reduced the incidence of symptomatic $\mathrm{BM}$, decreasing the cumulative risk at 1 year from $40.4 \%$ in the control group to $14.6 \%$ in the $\mathrm{PCl}$ group and prolonged the 1-year OS $(27 \%$ in $\mathrm{PCl}$ group versus $13 \%$ in the control arm, hazard ratio $[\mathrm{HR}]=0.68, \mathrm{p}=0.003)$. The trial, along with the previous meta-analysis, strengthened the role of $\mathrm{PCl}$ in the ED population and $\mathrm{PCl}$ became standard care in metastatic patients with its use being recommended in international guidelines [6,7]. Notably, an analysis of patterns of care in the USA showed high adherence to guidelines; namely, $98 \%$ of radiation oncologists recommended $\mathrm{PCI}$ for patients with ED-SCLC [8].

Over the past year the role of $\mathrm{PCI}$ in ED-SCLC has been readdressed [9] and conflicting results as far as OS is concerned have been reported. Takahashi and colleagues ran a phase III trial where patients were randomised, at the end of their standard first-line chemotherapy and in the absence of progression, to $\mathrm{PCl}$ or brain MRI monitoring. The study did not show any difference in terms of OS between the 2 
arms, with a median OS of 11.6 months (in the $\mathrm{PCl}$ arm) versus 13.7 months (in the observation arm) (HR = 1.27; 95\% confidence interval $[\mathrm{Cl}] 0.96-1.68, \mathrm{p}=0.94)$. The study was interrupted early and based on these findings, the role of $\mathrm{PCl}$ has been challenged and become controversial.

The aim of the present review is to critically analyze separately prospective and retrospective trials reporting results in terms of intracranial control and OS in EDSCLC patients with the intention of clarifying the role and finding a possible place for the use of $\mathrm{PCl}$ in this setting.

\section{MATERIALS AND METHODS}

An extensive review of the literature has been performed between April and September 2018 throughout PubMed. Articles reporting on "PCl in ED-SCLC" and "metastatic SCLC" have been identified and analyzed. In order to have a "modern view" of the literature, and avoid studies in which patients were treated with nonstandard radiotherapy (RT) doses or non-platinum containing regimes, only publications after 2000 have been considered. The following keywords have been entered to identify potential articles:[small cell lung cancer] AND [extensive disease] AND [PCI]. We found and screened 332 articles. Review articles, commentaries, case reports and abstracts have been excluded. Studies targeting limited stage (LS)SCLC or reporting on patients treated with concomitant therapy (systemic treatment 
and/or thoracic RT), and reports without clear comparison between $\mathrm{PCl}$ treated pts and observed population were excluded. The final reference list, based on originality and relevance to the broad scope of this review, contained 8 full-text articles, including prospective and retrospective trials that were included in the qualitative synthesis. Quantitative analysis was done on the final list of 6 articles. The flowchart is presented in Fig. 1

\section{RESULTS}

We have identified and included 6 studies in the review, 2 prospective $[5,9]$ and 4 retrospective [10-13]. All the retrospective series favor $\mathrm{PCl}$ in terms of $\mathrm{OS}$ and intracranial control. The 2 prospective trials report conflicting results with regards to OS, while both favor $\mathrm{PCl}$ in terms of local control.

Dose and fractionation vary slightly between the series (total $\mathrm{PCl}$ dose ranges from 24 to $30 \mathrm{~Gy}$ ), with 25 Gy in 10 fractions being the most common schedule (table 1 ).

Brain screening for BM at diagnosis was always performed in both prospective and retrospective trials with the exception of one prospective study [5] where screening for BM was performed only in $29 \%$ of patients. MRI was the preferred imaging modality. Computed tomography (CT) scan instead of MRI was used in a minority of cases and mostly due to MRI contraindication (pacemakers, artificial implants, etc). Only in one prospective study brain imaging was systematically performed after systemic treatment and before $\mathrm{PCl}$ [9]. In two studies there was no standardized protocol and only a few patients had brain imaging, either CT or MRI $[11,13]$. 
Information regarding brain imaging modalities before $\mathrm{PCl}$ is missing in two studies $[10,12]$.

Median follow up across studies was 23 months (range, 9-36).

Retrospective studies:

Within the retrospective studies, the largest one [10] used the American National Cancer Database and evaluated a total of 4257 SCLC metastatic patients (3784 not receiving $\mathrm{PCl}$ and 473 receiving $\mathrm{PCl}$ ). In this large cohort, patients treated between 2010 and 2012 with chemotherapy for metastatic SCLC and without BM have been analyzed. After propensity score matching on factors associated with receipt of $\mathrm{PCI}$ and OS, results in the overall cohort favored $\mathrm{PCl}$ with improved median survival (13.9 vs 11.1 months; $p<0.001)$, 1-year probability of survival $(61.2 \%$ vs $44 \%, p<0.001)$ and 2-year probability of survival (19.8\% vs $11.5 \%, \mathrm{p}<0.001)$. The benefit was confirmed even after excluding patients with less than 6 months or with less than 9 months survival (median survival for patients surviving at least 6 months: 14\% vs 11\%; median survival for patients surviving at least 9 months: $15 \%$ vs $13 \%$; $p$ 0.001).

More recently a publication from Ontario [11] reports results of 155 patients with EDSCLC and without baseline BM. Authors found a statistically significant difference in OS (HR 0.55; 95\% Cl 0.39-0.77; $\mathrm{p}=0.0005)$ and time to $\mathrm{BM}(\mathrm{HR} 0.40 ; 95 \% \mathrm{Cl} 0.23-$ $0.66 ; \mathrm{p}=0.0004$ ) with the use of $\mathrm{PCl}$. Median survival for the $\mathrm{PCl}$ and non- $\mathrm{PCl}$ groups was 13.5 and 8.5 months, respectively. Furthermore, the authors found a significant increase in 1 and 2-year OS in the PCl group (HR 0.41; 95\% Cl 0.29-0.57; p<.0001). The median time to develop BM was also found to be longer in the $\mathrm{PCl}$ group (23.8 
months vs 10.2 months) with an HR of 0.36 (95\% Cl 0.21-0.60; $p<.0001)$. A survival difference with $\mathrm{PCl}$ was observed in both patients that received post-chemotherapy brain imaging (HR 0.55; 95\% Cl 0.35-0.88; $\mathrm{p}=0.012)$ and those who did not (HR 0.48; 95\% Cl 0.29-0.77; $p=0.0025)$.

Furthermore Chen et al [12] reviewed 204 ED-SCLC patients who had any response after 4 to 6 cycles of chemotherapy. $\mathrm{PCl}$ was performed in 45 patients $(22.1 \%)$ and the remaining 159 (77.9\%) were observed. $\mathrm{PCl}$ significantly prolonged median OS (16.5 vs 12.6 months [HR $0.63,95 \% \mathrm{Cl} 0.41-0.96 ; \mathrm{p}=0.033]$ ). Also, the risk of developing $\mathrm{BM}$ was lower in the $\mathrm{PCl}$ group (HR 0.48; $95 \% \mathrm{Cl} 0.30-0.76 ; \mathrm{p}=0.001$ ), with 1-year incidence of $\mathrm{BM}$ of $17.1 \%$ vs $55.9 \%$ in the $\mathrm{PCl}$ and control group, respectively. $\mathrm{PCl}$ was a favorable independent predictor for $\mathrm{OS}$ in multivariate analysis. In this trial, brain imaging was mandatory prior to initial treatment.

Nicholls et al [13] reported their institutional experience and analyzed retrospectively patients with SCLC (both ED and LD) treated between January 2008 and December 2013. Of the 129 ED patient population, $13 \%$ received $\mathrm{PCl}$. Median OS in the EDSCLC cohort receiving PCl was 13.6 months compared to 5.6 months in patients not receiving such treatment $(p<0.001)$.

Prospective Studies

At present, there are only two prospective trials available. The first one is the EORTC study published in 2007 by Slotman et al [5]. The primary endpoint of the trial was the time to development of symptomatic BM, while secondary endpoints were survival, quality of life, toxic effects, and treatment costs. Cumulative risk of developing symptomatic BM within 1 year was $14.6 \%$ in the $\mathrm{PCl}$ group, compared to $40.4 \%$ in 
the control arm. Although OS was a secondary endpoint of the study, patients in the $\mathrm{PCl}$ group showed longer OS, with a median survival of 6.7 months compared to 5.4 months $(p=0.003)$. In this study, brain imaging was not mandatory before enrolment and not routinely performed during follow-up (unless patients presented symptoms suggestive of BM).

The second one was run by the Japan Clinical Oncology Group (JCOG): a prospective trial readdressing the role of PCI in ED-SCLC [9]. In this phase III trial, patients were randomised to $\mathrm{PCl}$ or observation with active monitoring. Conversely from the EORTC trial, the primary endpoint was OS, while time to BM was among secondary endpoints. This study did not demonstrate any difference in median OS between patients receiving $\mathrm{PCl}$ (11.6 months) and those assigned to the observation arm (13.7 months) (HR 1.27; 95\% Cl 0.96-1.68, p=0.94]. However, the cumulative incidence of $\mathrm{BM}$ at 6,12 and 18 months was lower in the $\mathrm{PCl}$ group compared to the observation group (15\%, 32.9\% and $40.1 \%$ vs $46.2 \%, 59 \%$ and $63 \%$, respectively).

In the current literature, reports on quality of life (QoL) and adverse events (AE) are inconsistent and reported only in three publications $[9,12,14]$. In the EORTC trial $[5$, 14], there was no statistically significant difference in global health status between the two groups from baseline to 9 months $(p=0.10)$. Most common side effects in the $\mathrm{PCl}$ group were hair loss and fatigue, that were significantly higher compared to control arm $(p<0.001)$. No significant differences were found between the study groups in role functioning $(p=0.17)$, cognitive functioning $(p=0.07)$ or emotional functioning $(p=0.18)$. In the JCOG trial [9], Mini-mental state examination (MMSE) scores were assessed at baseline, after 6 and 12 months, without significant differences between the two groups. Toxicity scores of grade 3 or higher were similar 
between the irradiated or observed group. The most frequent grade $3 \mathrm{AE}$ were anorexia ( $5 \%$ in the $\mathrm{PCl}$ group vs $2 \%$ in the observation cohort), malaise (3\% in the $\mathrm{PCl}$ group vs $1 \%$ in the observation cohort) and muscle weakness (1\% in the $\mathrm{PCl}$ group vs $5 \%$ in the observation cohort). Impact on QoL was not addressed in this trial. Chen et al [12] report acute and late adverse events in the irradiated population. Grade 3 or worse acute toxicities were overall low $(2.2 \%$ grade 3 headache, no grade 4 acute side effect, no grade 3 or 4 late effects). The most frequent grade 2 acute side effect was headache (6.7\%) and nausea or vomiting (4.4\%).

\section{DISCUSSION}

Due to the high likelihood to develop BM and the clinically relevant consequences that this event implies, the prevention and control of BM are of paramount importance in patients with SCLC. Consensus has been reached in the setting of LD-SCLC, where, according to international guidelines [6,7], $\mathrm{PCl}$ is part of standard treatment, providing a benefit in terms of survival and local control. However, in the ED-SCLC, the indication to deliver $\mathrm{PCl}$ is not universally established. So far, on the basis of the results from the EORTC trial [5], most guidelines $[6,7]$ recommended PCI for EDSCLC patients who achieved at least a partial response. However, after the publication of the JCOG trial [9], $\mathrm{PCl}$ in this setting has become more controversial due to the uncertainty of OS benefits and practice has been shifting. For example in the recent Impower 133 [15], a trial comparing in ED-SCLC chemotherapy vs chemotherapy plus Atezolizumab, $\mathrm{PCl}$ was optional and only $10 \%$ of the centers opted for it 
The EORTC study [5] was the cornerstone and first randomized trial. It showed that $\mathrm{PCl}$ reduces by two to three folds the risk to develop symptomatic $\mathrm{BM}$, and prolongs OS with improved 1-year survival reaching $30 \%$ in the treated population versus $13 \%$ in the non-PCl group. Key criticism to the EORTC trial is that patients were not systematically screened for BM (unless symptoms suggested so). This pragmatic approach may have led to treat with $\mathrm{PCl}$ some patients that already had $\mathrm{BM}$, and this could imply that the OS improvement could be due to the treatment of existing disease, and not to a true prophylactic effect. However, according to Hochstenbag et al [16], the proportion of patients with asymptomatic BM detected by follow-up MRI is rather small, about $15 \%$, and it seems unlikely that this small proportion can justify the observed OS benefit.

The JCOG study [9] found, in the first analysis based on 163 patients, no benefits in terms of $\mathrm{OS}$ with a median survival of 10.1 months in the $\mathrm{PCl}$ group compared with 15.1 months in the control group $(p=0.091)$. This finding led to the premature termination of the trial for futility. At the final analysis, with a longer follow-up of almost 12 months, the difference in median survival was less important (2 months difference vs 5 observed in the first analysis), leading the Authors to conclude that "prophylactic cranial irradiation is not essential" for patients with ED-SCLC if they are regularly assessed by MRI during follow-up, and treated for symptomatic metastases when required. Unlike the EORTC trial [5], all patients had brain MRI before the enrolment, and was performed during follow-up every 3 months for the first year, and every 6 months thereafter.

These results raise two important considerations. First, routine brain MRI imaging follow-up every 3 months would come with a considerable socioeconomic impact, which would not easily be applicable in clinical practice in the vast majority of the 
countries. Second, when comparing 1-year OS between the two trials, patients enrolled in the JCOG [9] had a much better survival in both the $\mathrm{PCl}$ arm and the control arm than in the EORTC trial [5] (53.6 \% vs $13.3 \%$ in the non-PCl arm, $48.4 \%$ vs $27.1 \%$ in the $\mathrm{PCl}$ arm). This may be partially explained by different genetics between Asian and Caucasian populations [17], but also by a possible selection bias. Indeed, the proportion of patients who were offered second-line chemotherapy was higher in the JCOG [9] compared to the EORTC [5] trial: within the JCOG [9], 40 patients in the observation arm (36\%) and 29 patients in the $\mathrm{PCl}$ group (26\%) were offered fourth-line chemotherapy. This is quite an exceptional event in SCLC patients and does not reflect daily practice.

In the cited retrospective series, as far as OS concerns, there's a clear advantage in favour of PCl.

It is worth discussing the systemic review and meta-analysis by Maeng et colleagues [18] , who used as primary endpoint OS. They analyzed 5 trials, 2 of them prospective. In pooled estimates, $\mathrm{PCl}$ did not statistically improve $\mathrm{OS}$ ( $\mathrm{HR}=0.82$; 95\%, Cl: $0.60,1.11 ; p=0.19)$. However, the $\mathrm{PCl}$ group had a significant advantage in 1-year survival (37.1\% versus $27.1 \%$; $95 \% \mathrm{Cl}$ : $0.80-0.95 ; \mathrm{p}=0.002)$, progressionfree survival $(\mathrm{HR}=0.83 ; 95 \% \mathrm{Cl}: 0.70-0.98 ; p=0.03)$ and decreased risk of $\mathrm{BM}$ $(\mathrm{HR}=0.34 ; 95 \% \mathrm{Cl}: 0.23-0.50 ; p<0.001)$ compared to the non-PCl group.

One more meta-analysis by Ge et colleagues [19] was recently published, assessing 14 papers, of which again only 2 were prospective. The results showed that $\mathrm{PCl}$ significantly improved overall survival $(\mathrm{HR}=0.57 ; 95 \%, \mathrm{Cl}: 0.47-0.69 ; p<0.001)$ and BM (RR $=0.47,95 \% \mathrm{Cl}: 0.33,0.69 ; p<0.01)$. However, this study had many flaws: an extremely heterogeneous population, the different $\mathrm{PCl}$ doses, and $\mathrm{PCl}$ timings. 
Development of symptomatic BM is responsible for low QoL, significant morbidity, including hospitalization, and a median survival of only 4 to 6 months [20-22]. The EORTC and JCOG trials [5, 9] have shown that PCI significantly reduces by 2- to 3fold the incidence of BM. In the Japanese trial [9] the cumulative incidence of BM at 6, 12 and 18 months was significantly higher in the observation arm. In this group, $83 \%$ of patients (compared to $46 \%$ in the $\mathrm{PCl}$ arm) needed radiotherapy; also in the EORTC trial, cranial irradiation was ultimately offered to $59 \%$ of patients with symptomatic $\mathrm{BM}$ in the non- $\mathrm{PCl}$ cohort vs $8.3 \%$ in the $\mathrm{PCl}$ cohort [5].

The results from retrospective series are in line with these findings. Chen et al [12] found a significantly increased BM-free survival $(p=0.002)$ with 1-year incidence of $\mathrm{BM}$ of $17 \%$ vs $56 \%$ in the $\mathrm{PCl}$ and non- $\mathrm{PCl}$ group, respectively. The median time for the development of BM was also found to be longer in the PCl group (23.8 months vs 10.2 months) by the Ontario study [11].

Although the CALGB 30504 study [23] was not included in our final list because of the presence of a possible confounding factor (i.e. the use of an anti-angiogenic tyrosine kinase inhibitor), we find it is worth taking into consideration the post-hoc unplanned analysis. This trial was a phase II randomized trial of sunitinib vs placebo in EDSCLC patients responding to platinum-based chemotherapy. Brain imaging was required at pre-enrollment. $\mathrm{PCl}$ was delivered at the discretion of the treating physician. Results showed a trend for improved progression-free survival (PFS) and OS in patients receiving $\mathrm{PCl}$ and sunitinib, with a quite remarkable central nervous system progression in the non-PCI group (27\% vs $12 \%, p=0.05)$.

Thus, the burden of brain failure is relevant and must be considered, for both the patients QoL that deteriorates with such an event [24] and the balance of cost/benefit. 
Over the past decade concerns regarding the possible decline of the neurocognitive functions (NCF) in patients treated with $\mathrm{PCl}$ have surfaced. However, there is no robust data to demonstrate any difference in NCF with or without $\mathrm{PCl}$ [25]. In a review by Tallet et al [26] on NCF in patients, after whole-brain radiotherapy, (patients were offered PCl or therapeutic WBRT), most studies assessing NCF in the setting of $\mathrm{PCl}$ showed a very low incidence of neurocognitive impairment at one year. MMSE was the only parameter assessed within the Japanese trial [9], and it did not show any difference after 12 and 24 months. Furthermore, most patients already have abnormal neuropsychology testing after chemotherapy and before $\mathrm{PCl}$, stressing the importance to assess the chemotherapy impact on NCF. QoL was tested in the EORTC trial [5] and the impact of irradiation on functioning scales was moderate. Finally, neuro-toxicity induced by cranial irradiation may be reduced by hippocampal sparing. A prospective study suggested a potential benefit of this technique in limiting the neuropsychological sequelae of brain radiation for patients treated with $\mathrm{PCl}$ for LD-SCLC [27]. However, the safety of hippocampal-avoidance $\mathrm{PCl}$ still needs to be further validated in terms of risk of brain failure in the SCLC population.

To comment on doses and schedules, wide variation is seen among studies with doses ranging from 20 to 30 Gy resulting in different biologically equivalent doses (up to 39 Gy with an $\alpha / \beta$ of 10 Gy). Twenty-five Gy in 10 fractions is nowadays the standard. Higher dose do not translate into higher local control, and are associated with a possible negative impact on NCF [28]. The use of higher doses and dose per fraction in the past could, therefore, have had an impact on toxicity without any benefits in terms of disease control. 
International guidelines, such as Version 1.2019 National Comprehensive Cancer Network (NCCN) guidelines [29] suggest to consider PCI or MRI brain surveillance in ED-SCLC with complete or partial response to first line chemotherapy.

\section{CONCLUSIONS}

Despite contradictory findings about a possible OS advantage in favour of $\mathrm{PCI}$ in the literature and the need to run further prospective trials, our review has further confirmed that $\mathrm{PCl}$ plays a role in reducing the incidence of developing $\mathrm{BM}$ in EDSCLC patients responding to first-line platinum-based chemotherapy.

We would strongly encourage oncologists and radiation oncologists to consider all the factors and evidence we have gathered in this review, and to share them openly with the patients in order to make an informed choice based on the evidences rather than personal belief 


\section{REFERENCES}

Papers of particular interest, published recently, have been highlighted as:

*of importance, $* *$ outstanding importance.

[1] Goldstraw P, Crowley J, Chansky K et al. The IASLC Lung Cancer Staging Project: proposals for the revision of the TNM stage groupings in the forthcoming (seventh) edition of the TNM Classification of malignant tumours. Journal of thoracic oncology : official publication of the International Association for the Study of Lung Cancer 2007; 2:706-714.

[2] van Meerbeeck JP, Fennell DA, De Ruysscher DK. Small-cell lung cancer. Lancet 2011; 378:17411755.

[3] Jackson DV, Jr., Richards F, 2nd, Cooper MR et al. Prophylactic cranial irradiation in small cell carcinoma of the lung. A randomized study. Jama 1977; 237:2730-2733.

[4] Auperin A, Arriagada R, Pignon JP et al. Prophylactic cranial irradiation for patients with small-cell lung cancer in complete remission. Prophylactic Cranial Irradiation Overview Collaborative Group. The New England journal of medicine 1999; 341:476-484.

[5] Slotman B, Faivre-Finn C, Kramer G et al. Prophylactic cranial irradiation in extensive small-cell lung cancer. The New England journal of medicine 2007; 357:664-672.

[6] Rudin CM, Ismaila N, Hann CL et al. Treatment of Small-Cell Lung Cancer: American Society of Clinical Oncology Endorsement of the American College of Chest Physicians Guideline. Journal of clinical oncology : official journal of the American Society of Clinical Oncology 2015; 33:4106-4111.

[7] Fruh M, De Ruysscher D, Popat S et al. Small-cell lung cancer (SCLC): ESMO Clinical Practice Guidelines for diagnosis, treatment and follow-up. Annals of oncology : official journal of the European Society for Medical Oncology 2013; 24 Suppl 6:vi99-105.

[8] Jain A, Luo J, Chen Y et al. Current Patterns of Care for Patients with Extensive-Stage SCLC: Survey of U.S. Radiation Oncologists on Their Recommendations Regarding Prophylactic Cranial Irradiation. Journal of thoracic oncology : official publication of the International Association for the Study of Lung Cancer 2016; 11:1305-1310.

[9] Takahashi T, Yamanaka T, Seto T et al. Prophylactic cranial irradiation versus observation in patients with extensive-disease small-cell lung cancer: a multicentre, randomised, open-label, phase 3 trial. The Lancet. Oncology 2017; 18:663-671.

[10] Sharma S, McMillan MT, Doucette A et al. Effect of Prophylactic Cranial Irradiation on Overall Survival in Metastatic Small-Cell Lung Cancer: A Propensity Score-Matched Analysis. Clinical lung cancer 2018; 19:260-269 e263.

[11] Bang A, Kendal WS, Laurie SA et al. Prophylactic Cranial Irradiation in Extensive Stage Small Cell Lung Cancer: Outcomes at a Comprehensive Cancer Centre. International journal of radiation oncology, biology, physics 2018; 101:1133-1140.

[12] Chen Y, Li J, Hu Y et al. Prophylactic cranial irradiation could improve overall survival in patients with extensive small cell lung cancer : A retrospective study. Strahlenther Onkol 2016; 192:905-912. Single institution publication showing clear advantage to sue $\mathrm{PCl}$ in term of survival benefit.

*[13] Nicholls L, Keir GJ, Murphy MA et al. Prophylactic cranial irradiation in small cell lung cancer: A single institution experience. Asia-Pacific journal of clinical oncology 2016; 12:415-420.

[14] Slotman BJ, Mauer ME, Bottomley A et al. Prophylactic cranial irradiation in extensive disease small-cell lung cancer: short-term health-related quality of life and patient reported symptoms: results of an international Phase III randomized controlled trial by the EORTC Radiation Oncology and Lung Cancer Groups. Journal of clinical oncology : official journal of the American Society of Clinical Oncology 2009; 27:78-84. 
[15] Horn L, Mansfield AS, Szczesna A et al. First-Line Atezolizumab plus Chemotherapy in ExtensiveStage Small-Cell Lung Cancer. The New England journal of medicine 2018; 379:2220-2229.

[16] Hochstenbag MM, Twijnstra A, Wilmink JT et al. Asymptomatic brain metastases (BM) in small cell lung cancer (SCLC): MR-imaging is useful at initial diagnosis. Journal of neuro-oncology 2000; 48:243-248.

[17] Zhou W, Christiani DC. East meets West: ethnic differences in epidemiology and clinical behaviors of lung cancer between East Asians and Caucasians. Chinese journal of cancer 2011; 30:287-292.

This publication confirmed the role of $\mathrm{PCl}$ in term of intracranial activity. As it failed to show an overal survival benefit, this publication hightlighted once more the controversy about $\mathrm{PCl}$ in this setting.

** [18] Maeng CH, Song JU, Shim SR, Lee J. The Role of Prophylactic Cranial Irradiation in Patients With Extensive Stage Small Cell Lung Cancer: A Systematic Review and Meta-Analysis. Journal of thoracic oncology : official publication of the International Association for the Study of Lung Cancer 2018; 13:840-848.

This publication highlithed once more the importance and the role of $\mathrm{PCl}$ in ED-SCLC in both reduction the incidence of brain metastases and improving overal survival.

**[19] Ge W, Xu H, Yan Y, Cao D. The effects of prophylactic cranial irradiation versus control on survival of patients with extensive-stage small-cell lung cancer: a meta-analysis of 14 trials. Radiation oncology 2018; 13:155.

[20] Felletti R, Souhami RL, Spiro SG et al. Social consequences of brain or liver relapse in small cell carcinoma of the bronchus. Radiotherapy and oncology : journal of the European Society for Therapeutic Radiology and Oncology 1985; 4:335-339.

[21] Lee JJ, Bekele BN, Zhou X et al. Decision analysis for prophylactic cranial irradiation for patients with small-cell lung cancer. Journal of clinical oncology : official journal of the American Society of Clinical Oncology 2006; 24:3597-3603.

[22] Gregor A, Cull A, Stephens RJ et al. Prophylactic cranial irradiation is indicated following complete response to induction therapy in small cell lung cancer: results of a multicentre randomised trial. United Kingdom Coordinating Committee for Cancer Research (UKCCCR) and the European Organization for Research and Treatment of Cancer (EORTC). European journal of cancer 1997; 33:1752-1758.

[23] Salama JK, Gu L, Wang X et al. Positive Interaction between Prophylactic Cranial Irradiation and Maintenance Sunitinib for Untreated Extensive-Stage Small Cell Lung Cancer Patients After Standard Chemotherapy: A Secondary Analysis of CALGB 30504 (ALLIANCE). Journal of thoracic oncology : official publication of the International Association for the Study of Lung Cancer 2016; 11:361-369. [24] Le Rhun E, Taillibert S, Blonski M et al. [Supportive care, cognition and quality of life in brain metastases]. Cancer radiotherapie : journal de la Societe francaise de radiotherapie oncologique $2015 ; 19: 55-60$.

[25] Grosshans DR, Meyers CA, Allen PK et al. Neurocognitive function in patients with small cell lung cancer : effect of prophylactic cranial irradiation. Cancer 2008; 112:589-595.

[26] Tallet AV, Azria D, Barlesi F et al. Neurocognitive function impairment after whole brain radiotherapy for brain metastases: actual assessment. Radiation oncology 2012; 7:77.

[27] Redmond KJ, Hales RK, Anderson-Keightly $\mathrm{H}$ et al. Prospective Study of Hippocampal-Sparing Prophylactic Cranial Irradiation in Limited-Stage Small Cell Lung Cancer. International journal of radiation oncology, biology, physics 2017; 98:603-611.

[28] Le Pechoux C, Dunant A, Senan S et al. Standard-dose versus higher-dose prophylactic cranial irradiation $(\mathrm{PCl})$ in patients with limited-stage small-cell lung cancer in complete remission after chemotherapy and thoracic radiotherapy (PCI 99-01, EORTC 22003-08004, RTOG 0212, and IFCT 9901): a randomised clinical trial. The Lancet. Oncology 2009; 10:467-474.

[29] Network NCC. Small Cell Lung Cancer (Version 2.2018). 